\title{
Research on Spread Rules of Network Opinions of Public Incident
}

\author{
Lin longxian \\ Zhejiang Police College , Hangzhou City Zhejiang Province , 310053,China
}

Keywords: Network opinions, Public incident, Spread rules

\begin{abstract}
With the popularity of the internet, the number of internet users in China continues to rise. Both the dissemination content and the dissemination speed of the network public opinion are rising rapidly in recent years. The governments at all levels should attach great importance to the transmission of network opinions of public incident. This paper discusses the life cycle of online public opinion, divides the spread of network opinions of public incident into three stages, which are the growth stage, the development period and the decline stage, and explores the rules in each stage to provide some references for the relevant researchers.
\end{abstract}

\section{Introduction}

Internet public opinion is a kind of network public opinion which is popular on the internet. It is a manifestation of social public opinion, which has strong influence and tendentious attitude towards certain hot spots and focus problems in real life. Network public opinion is usually formed through the channels of news commentary, micro-blog, WeChat and so on. With the rapid development of new media in our country, the network has gradually become an important carrier to reflect social public opinion. Since the birth of the Internet, globalization and low threshold have become its main characteristics. Under the mapping of these features, network public opinion is easy to be labeled and signed. Compared to the traditional public opinion, the network public opinion is more arbitrary, the scope of communication is broader, affecting more audiences, the audience is also more interactive participation. The fermentation time of public opinion is also shorter than the traditional state. These characteristics make the network public opinion become more and more volatile, and control is difficult. The virtual characteristics of the Internet also make the publication and evolution of the network public opinion more casual. There is hardly any strong restraint on the punishment of the existing laws, and more of them are condemned on the moral level. But the fact is the network public opinion when the thing person, probably because of this with an overwhelming public opinion widely caused great mental stress to appear tragic consequences for everyone to see. These examples have appeared in various news reports in recent years. We should analyze and summarize the basic rules of emergency network public opinion evolution to construct Chinese emergency network public opinion supervision and provide theoretical support and practical guidance for the government scientific emergency network public opinion.

\section{Life Cycle of Network Opinions of Public Incident}

The concept of life cycle stems from the biological field, mainly referring to a series of stages or changes in the form or function of organisms during the process of life evolution. Essentially, it refers to the whole stage and process of an organism experiencing its birth, birth, growth, aging and dying. Different information in different environments, or in a different evolution process of different flow of information, but all follow a unified rule. The information ecosystem of information is experiencing the development period, the growth period, the optimization phase and decline phase evolution process, a life cycle of information now a complete, successive. The trend line is basically the same, that in the event of an emergency post. The amount of reading and comments will be a 
significant increase in the process, and reach the highest degree of concern events. Since then, with the event gradually subsides, the concern of the network gradually decays. According to the basic evolution trend of this process, the network public opinion of emergencies is divided into three stages: the growth stage (A-B section), the development period (B-C section) and the decline stage (C-D section), which is shown in Figure 1 . After entering the growth stage, the visits and the rapidly rising concern in the event of emergency network public opinion soon. Like the two times curve of the variation trend, the growth period enters the mature period, Internet traffic and comments reached the maximum value, the network public opinion strength and heat also enter the peak, and then with the event subsided. The network public opinion also enters a recession, and the netizens attention will gradually weaken.

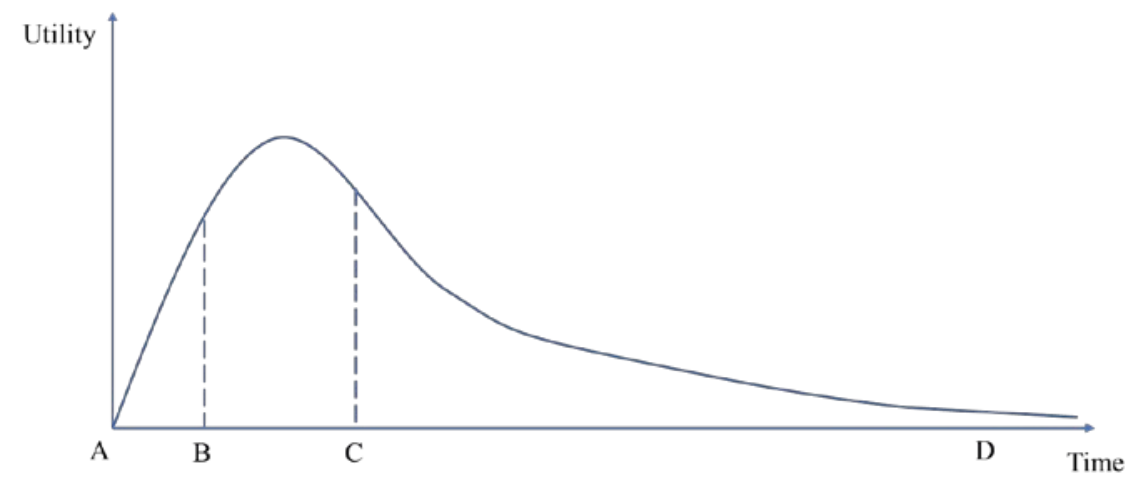

Figure 1. Figure of life cycle of network opinions of public incident

\section{Spread Rules of Network Opinions of Public Incident}

Occurrence Rules of Network Opinions of Public Incident.The internet can focus the attention of events to a specific point, forming the focus of attention of network public opinion. Some of the original not complicated sporadic ordinary events, through the superposition and focus of the Internet after the event has been enlarged, and has become a common concern of the seriousness of the network group events. On network group event plays a focusing amplification effect, and the birth of emergency network public opinion. Because of the imbalance of social and economic development and the diversification of social interests and the diversity of ideas of the public, the complexity and variability of social contradictions and social psychology have been increased. The unfairness of some social groups or the public, especially the social vulnerable groups. It is easy for them to focus their attention on the government and so on. Social deep-seated contradictions will accumulate during time aggregation and network media deliberately rendering amplification, so that the public mood found agglomeration focus and breakthrough point. All the social discontent and personal emotions will lead to accumulation of emotions because a focused incident occurred, and the outbreak of Internet public opinion in time. Especially in the network environment, the network communication in low cost, easy access conditions and network popularity gathered speed, and the spread of the scale and scope, the superimposed focusing effect of network public opinion development is obvious. Once a social event occurs, the public spontaneously causes events, causes, or attacks on society or government. They use the Internet to exaggerate, post, and make web reviews and hype, triggering widespread emotional convergence and focus. When the emotion is superimposed to a certain limit, the unexpected public opinion will break out.

Diffusion Rules of Network Opinions of Public Incident.After the emergency network public opinion, the substantive network of public opinion breaks out. Public opinions begin to enter the diffusion stage. Internet public opinion has realized the linear model of mutual communication among individuals. Linear progressive mode only on the line of emergency network public opinion in the process of diffusion and diffusion description, because of the formation of network public opinion is a structure and the complex process of external and internal factors can lead to network public opinion diffusion process and the shape of the difference. Although in the Internet world of public microphones, the issue of online public opinion of emergencies is diverse and fragmented, and the 
diffusion and change of public opinion are different. Unexpected events are very likely to produce ripple effect. Whenever the accident occurs, the event and its derivative events want to throw a stone into the water and arouse waves in the water. It causes ripples and forms a wider range of online public opinion, which has a greater negative impact on the outside. In the study of public opinion, ripple effect is an important mode of public opinion communication and diffusion. Because of the ubiquity and convenience of the internet, the public will form a comment or an emotional overflow of events or events beyond the Internet because of the ubiquity and convenience of the internet. With the continuous proliferation of public opinion sources, the scope of influence is more and more wide, and the public begin to concern themselves from the development of the event itself to the deep-seated causes of events. The public has pointed at some institutional or social levels, triggering wider and deeper public opinion. As public opinion, attitudes and opinions move closer to each other, the network public opinion fluctuations are magnified. Public opinion goes from the original disorderly comment to orderly condemnation.

Decline Rules of Network Opinions of Public Incident. The conflict rule of the network public opinion of the emergency event mainly refers to the behavior or phenomenon that the public opinion, such as the government, the media and so on. The internet public opinion reduces and disappears the network public opinion. Emergency network public opinion in the evolution process, the main event of the stakeholders, the government, the media and the public four main subjects of public opinion, and the four-main public opinion because of their own interests' preference or mood, play different roles in the evolution of network public opinion for emergencies. According to the network public opinion evolution in the role, can be divided into driving behavior model and block behavior model, interaction of them, in the evolution process of emergency network public opinion in the shift, promoting the development of network public opinion. In the later stage of emergency network public opinion evolution, the main force is block effect, mainly through control reports, prevention and control of the government intervention, shielding the event message or delete posts and other behavior of the event, to weaken the diffusion force of emergency network public opinion, reduce network public opinion. The government's control mechanism for online public opinion, its greatest advantage is that in the event of an emergency. Many negative public opinions in the network have been timely control. The development of public opinion to a certain extent, the government, media and other public opinion will hinder the development of intervention. Under the influence of this conflict resistance, the development trend of network public opinion began to weaken, and with the increase of the conflict resistance of online public opinion, the development of network public opinion tends to decay, and even disappear.

\section{Countermeasures of Network Opinions of Public Incident Based on Spread Rules}

Establish Public Opinion Institutions. Only understanding the public opinion behind the online public opinion can we better deal with it. Due to the sudden, rapid and complex content of online public opinion, the difficulty of monitoring is increased. Therefore, the government should set up a special monitoring body, the understanding of public opinion through the monitoring data and survey results, and through the massive data to supplement and confirm, analysis and identification, timely warning of crisis. Governments at all levels are setting up teams to monitor, analyze and respond to the Internet public opinion, and set up related duty system and emergency response mechanism. In addition, many news organizations, including the official media, are slowly setting up special public opinion analysis, judgment, evaluation and publishing mechanism. The function of public opinion detection and monitoring system, fundamentally speaking, the monitoring of network public opinion requires real-time, comprehensive and accurate. Public opinion was earlier, leaving in coping with the greater room for development, and the network public opinion is often explosive, transient opportunities, once missed the best time will be too late to regret. To ensure the timeliness, accuracy and comprehensiveness of public opinion monitoring, public opinion monitoring system is the most reliable way is to use automated software system to eliminate the disadvantages, rely on artificial way, and automatically finishing will generate reports, public opinion and government work closely. 
Enhance Dynamic Response Speed. The occurrence of network public opinion of emergencies often has the characteristics of serious situation and explosive news. Especially in the current high-speed development of the Internet, a mobile phone can instantly spread an event throughout the country. The event of emergencies if not promptly resolved, which is likely to make the event deteriorated rapidly, make it difficult to clean up the situation, so promoting the local government emergency response to public opinion is a pressing matter of the moment is fast speed, one of the effective methods to resolve the situation to expand the. Online reports quickly attracted a lot of thread, the incident in a few days rapidly warming fermentation, and in the next few days continues to heat up. The local government found that the network of public opinion quickly after the composition of the working group rushed to the local investigation of things, and timely survey results will be announced to the public, evidence, witness testimony is sufficient, the truth is true, the network of public opinion quickly subsided, it is because the local government reflects the prompt, very secure will be transferred to the line of sight of the masses the true situation, not only did not cause large public confusion, and even appeared in the public opinion reversal, this incident quickly subsided, can be said that the local government because of timely response, successfully resolve the crisis of network public opinion. The relevant departments quickly find the network public opinion demands and timely response, which is the key to the success of online public opinion of unexpected events.

Construct Early Warning Mechanisms.Local governments should keep pace with the times, face the new challenges brought by the development of the times, and study the laws of the development of online public opinion seriously, so that they can take preventive measures and not be caught unprepared. The use of advanced information technology, establish and improve the network public opinion warning and monitoring system to achieve early warning, the establishment of public opinion monitoring platform dedicated from the service network of public opinion investigation supervision work, to discover the potential, have the potential to generate online public opinion information, which can resolve the prospective emergencies may occur the crisis. For the public opinion has been formed after the event to do a good job of warning, we should monitor the development of public opinion, analyze the causes and provide theoretical basis for its solution. The local government, leading cadres and staff as the staff directly facing the crisis, the development trend of events the relationship between coping ability level, if properly handled, it will greatly increase the possibility of resolving the crisis. Each department can carry out the normal training and enhance the ability to respond to public opinion, summarize the experience and lessons and flexible ways to improve the ability to guide all kinds of civil servants at all levels of public opinion sensitive ability, public opinion, media communication skills, so that they can respond in the face of network public opinion events.

\section{Conclusion}

Dealing with the network public opinion under the background of unexpected events is helpful to minimize the impact of unexpected incidents in society. Only the local government deeply understands the importance of network public opinion management, clarifies the dissemination characteristics of network opinions of public incidents and establishes a set of effective network public opinion response mechanisms, can the government scientifically and effectively response to the crisis of the network public opinionand promote the harmonious development of society.

\section{References}

[1] Sun Ruiying, Wang Xu. Empirical Studies of Network Public Opinion Spread during Emergencies [J]. Journal of Modern Information, 2016, 36(12): 65-72+77.

[2] Hou Lei. A Research on the Online Public Opinion Control Mechanism of Emergency in the Big Data Era [J]. Public Administration \& Law, 2016, (10): 67-72. 
[3] Wang Xu, Sun Ruiying. Research about Online Public Opinion Spread during Emergencies Based on SocialNetwork Analysis-A Case Study of the Wei Zexi [J]. Information Science, 2017, 35(3): 87-92.

[4] Li Zhaocui. Research on Public Opinion Propagation and Early Warning Model of Emergency Network [J]. Computer \& Telecommunication, 2016(10): 30-32. 